\title{
Pensamiento sistémico en el modelo de resolución de problemas en estudiantes de tercer grado de secundaria
}

\section{Systemic Thinking in the Problem Solving Model in Third Grade High School Students}

\author{
Montilla-García, Henrry [(1) 0000-0001-8332-2643]। \\ 'Universidad Nacional de San Martín, Tarapoto, Perú \\ $\triangle$ henrrymontilla@gmail.com
}

Recibido: 23/10/2021;

Aceptado: 26/II/202I;

Publicado: 20/0I/2022

Resumen: Excluir al pensamiento sistémico de la enseñanza y el aprendizaje de la matemática en el enfoque del modelo constructivista, cuyos elementos no evidencian una articulación con la resolución de problemas, no permite logros de aprendizaje y ocasiona la carencia de un modelo basado en el pensamiento sistémico para la resolución de problemas complejos de matemática. El estudio tuvo como objetivo principal analizar la relación entre el pensamiento sistémico y el modelo de resolución de problemas en los estudiantes del tercer grado de secundaria de la Institución Educativa "Santa Rosa" de Tarapoto. La investigación fue de tipo básica, nivel correlacional, con diseño no experimental y descriptivo. La muestra censal estuvo conformada por 163 estudiantes. Los datos se recolectaron por medio de dos rúbricas de evaluación aplicados en la observación del desempeño de los estudiantes al participar de la resolución de problemas matemáticos en una sesión de aprendizaje. Los resultados indicaron que la medida tau-b de Kendall de la relación entre el pensamiento sistémico y el modelo de resolución de problemas fue de 0,9l8; para una prueba bilateral con p-valor $=0,000<0,05$. Se concluye que existe relación muy alta y positiva entre el pensamiento sistémico y el modelo de resolución de problemas.

Palabras clave: aprendizaje; enseñanza; general; matemática; sistemas; teoría

Abstract: To exclude systemic thinking from teaching and learning mathematics in the approach of the constructivist model, whose elements do not show an articulation with problem solving, is enabling low learning achievement and the lack of a systemic thinking-based model for solving complex mathematical problems. The main objective of the study was to analyze the relationship between systemic thinking and the problem-solving model in students in the third grade of the "Santa Rosa" Educational Institution of Tarapoto. The research was of basic type, correlational level, with nonexperimental and descriptive design. The census sample consisted of 163 students. The data were collected through two assessment rubrics applied in observing the performance of students by participating in solving mathematical problems in a learning session. The results indicated that Kendall's taub-b measure of the relationship between systemic thinking and the problem-solving model was 0.918 ; for a bilateral test with $p$-value $=0,000<0,05$; therefore. We concluded that there is a very high and positive relationship between systemic thinking and the problem-solving model.

Keywords: general; learning; mathematics; systems; teaching; theory

*El artículo es el resultado de un trabajo de investigación para la obtención del título profesional en Ingeniería de Sistemas e Informática en la Universidad Nacional de San Martín, Perú.

Cómo citar / Citation: Montilla-García, H. (2022). Pensamiento sistémico en el modelo de resolución de problemas en estudiantes de tercer grado de secundaria. Revista científica de sistemas e informática, 2(I), el62. https://doi.org/I0.5I252/rcsi.v2il.I62 


\section{Introducción}

La teoría general de sistemas enfocada en el desarrollo del pensamiento sistémico, insiste en adoptar nuevos modelos teóricos, metodológicos y epistemológicos que permitan la elaboración de propuestas didácticas acordes con la realidad de la enseñanza y el aprendizaje, y posibiliten el diseño y la puesta en práctica de modelos que permitan una intervención educativa más eficaz; lo que implicaría una línea consciente y sistémica de componentes cognitivos, metacognitivos y socioafectivos para la mejora de las habilidades de aprendizaje y del nivel de procesamiento y manejo de la información, a fin de facilitar el logro de las tareas por parte de los estudiantes (Corcino-Barrueta et al., 202I).

La necesidad de incorporar al pensamiento sistémico en el desarrollo de problemas en la complejidad, no puede más que imponerse de manera progresiva en un camino en que surgen límites, insuficiencias y carencias del pensamiento simplificante, propios de las estrategias de resolución de problemas matemáticos en el enfoque del constructivismo; es decir, las condiciones en las cuales no es posible dejar de considerar el desafío de lo complejo. En este escenario, se ejercita un pensamiento dialogante y negociador con lo real, que integra los modos simplificadores de pensar; rechazando las consecuencias mutilantes, reduccionistas y unidimensionales de las situaciones problemas (Barragán Moyano et al., 20I8).

El presente estudio identificó como problema, una inadecuada aplicación del modelo de resolución de problemas matemáticos a causa de la exclusión del pensamiento sistémico de la enseñanza y aprendizaje, en el que sus elementos tienen muy poca relación con el constructivismo; contribuyendo al bajo logro de aprendizajes en los estudiantes del tercer grado de secundaria de la I. E. "Santa Rosa" - Tarapoto. Al respecto, Corcino-Barrueta et al. (202I) indican que la educación debe modificar sus contenidos y aportes curriculares, asumiendo una metodología didáctica dentro de un modelo sistémico, con una visión integradora que relacione las partes en una totalidad y valore su evolución en su entorno.

En el desarrollo de la investigación, el escaso hábito de resolver problemas y el desconocimiento de los contenidos matemáticos básicos para operativizar estrategias algorítmicas de solución; fueron las limitaciones evidenciadas en los estudiantes, que dificultaron la observación y recolección de los datos. Al respecto, Donoso Osorio et al. (2020) explican que la resolución de un problema es un proceso con una serie de pasos en complejidad progresiva durante su desarrollo, en el que el estudiante integra la información textual con conocimientos matemáticos necesarios al contexto problemático que permite la solución; de lo contrario se hace dificultoso al resolver el problema.

Los objetivos de la investigación fueron: analizar y medir la relación entre el pensamiento sistémico y el modelo de resolución de problemas, determinar las causas de los bajos logros en la resolución de problemas con el modelo constructivista; diseñar un modelo basado en el pensamiento sistémico para la resolución de problemas complejos de matemática.

Los resultados de la investigación confirmaron la existencia de una relación muy alta y positiva entre el pensamiento sistémico y la resolución de problemas. Su importancia derivó en el planteamiento de un modelo de enseñanza-aprendizaje basado en la concreción de los niveles de dominio del pensamiento sistémico y la resolución de problema complejos de matemática, empleándose para el cual Vensim PLE 7.3.5, como software de simulación dinámica. 


\section{Materiales y métodos}

La investigación desarrollada fue de tipo básica, nivel correlacional, con diseño no experimental y descriptiva. La población en su totalidad fue tomada como muestra censal y constituida por 163 estudiantes.

Se tomó como referencia el marco teórico de Villa Sánchez \& Poblete Ruiz (2017) para elaborar las rúbricas de evaluación del pensamiento sistémico y de la resolución de problemas que permitieron la medición de sus niveles de dominio, respectivos; las cuales fueron sometidas al criterio de expertos; antes de su aplicación. La recolección de los datos se realizó al observar el desempeño en la resolución de problemas de matemática de cada estudiante de tercer grado de secundaria, con edades desde los 14 hasta los 17 años de la I. E. "Santa Rosa" - Tarapoto, en el año 2019.

Para lograr el análisis y la medición de la relación entre el pensamiento sistémico y el modelo de resolución de problemas, se han seguido los siguientes pasos: aplicación de las dos rúbricas de evaluación, procesamiento de los datos recolectados empleando Excel 2019 y SPSS 23, cálculo de coeficientes de correlación " $t$ " (tau b de Kendall) entre las variables estudiadas y sus niveles correspondientes; y análisis, presentación y explicación de resultados basados en los valores de coeficientes de correlación "t" obtenidos. Sáenz López \& Tamez González (2018) refieren que el coeficiente de correlación tau b de Kendall es una medida no paramétrica de asociación para variables ordinales y muestras pequeñas.

Para determinar las causas académicas, materiales y metodológicas de los bajos logros de aprendizaje en la resolución de problemas con el modelo constructivista, se realizaron los pasos: observación de la situación actual, aplicación del diagrama de Ishikawa para obtener el diagnóstico; y sistematización de las causas. Gutiérrez Verde et al. (2020) explican que el diagrama de Ishikawa o causa-efecto, representa gráficamente la relación que existe entre algún efecto y el conjunto de factores causantes que intervienen o influencian en una determinada característica.

Para diseñar el modelo basado en el pensamiento sistémico en favor de la resolución de problemas complejos de matemática se desarrollaron los pasos: revisión y análisis de las propuestas de Villa Sánchez \& Poblete Ruiz (2017), y de la propuesta constructiva del Ministerio de Educación (2016), utilización del diagnóstico de causas de bajos logros en resolución de problemas, y elaboración del modelo con dinámica de sistemas fundamentado en Gil Vera \& Gil Vera (2017) como técnica de modelado de los problemas complejos de matemática; y desarrollo de los modelos empleando el software Vensim PLE 7.3.5. 


\section{Resultados}

Tabla I. Resultados de la correlación entre las variables.

\begin{tabular}{|c|c|}
\hline Variables & Correlación \\
\hline Pensamiento sistémico y resolución de problemas. & Muy alta y positiva \\
\hline Primer y segundo nivel de dominio del pensamiento sistémico. & Muy alta y positiva \\
\hline $\begin{array}{c}\text { Pegundo y tercer nivel de dominio del pensamiento sistémico. } \\
\text { Segundo y tercer nivel de dominio de la resolución de problemas. } \\
\text { problemas. }\end{array}$ & Muy alta y positiva \\
\hline $\begin{array}{c}\text { Primer nivel de dominio del pensamiento sistémico y la resolución de } \\
\text { problemas. }\end{array}$ & Moderada y positiva \\
\hline $\begin{array}{c}\text { Segundo nivel de dominio del pensamiento sistémico y la resolución de } \\
\text { problemas. }\end{array}$ & Muy alta y positiva \\
\hline $\begin{array}{c}\text { Tercer nivel de dominio del pensamiento sistémico y la resolución de } \\
\text { problem }\end{array}$ & Alta y positiva \\
\hline
\end{tabular}

Los resultados de la correlación entre las variables son: muy alta y positiva entre el pensamiento sistémico y la resolución de problemas, muy alta y positiva entre el primer y segundo nivel de dominio del pensamiento sistémico, muy alta y positiva entre el segundo y tercer nivel de dominio del pensamiento sistémico, alta y positiva entre el primer y segundo nivel de dominio de la resolución de problemas.

Además de correlaciones alta y positiva entre el segundo y tercer nivel de dominio de la resolución de problemas, moderada y positiva entre el primer nivel de dominio del pensamiento sistémico y la resolución de problemas, muy alta y positiva entre el segundo nivel de dominio del pensamiento sistémico y la resolución de problemas, alta y positiva entre el tercer nivel de dominio del pensamiento sistémico y la resolución de problemas.

Tabla 2. Resultados de la medición de la correlación entre las variables.

\begin{tabular}{|c|c|}
\hline Variables & Valor "t" \\
\hline Pensamiento sistémico y resolución de problemas. & $0,9 / 8$ \\
\hline Primer y segundo nivel de dominio del pensamiento sistémico. & $\mathrm{I}$ \\
\hline Segundo y tercer nivel de dominio del pensamiento sistémico. & $\mathrm{I}$ \\
\hline Primer y segundo nivel de dominio de la resolución de problemas. & $0,83 \mathrm{I}$ \\
\hline Segundo y tercer nivel de dominio de la resolución de problemas. & 0,938 \\
\hline Primer nivel de dominio del pensamiento sistémico y la resolución de problemas. & 0,646 \\
\hline Segundo nivel de dominio del pensamiento sistémico y la resolución de problemas. & 0,934 \\
\hline Tercer nivel de dominio del pensamiento sistémico y la resolución de problemas. & 0,876 \\
\hline
\end{tabular}

Los resultados de los valores de los coeficientes "t" (tau b de Kendall) de la medición de la correlación entre las variables son: 0,918 entre el pensamiento sistémico y la resolución de problemas, I entre el primer y segundo nivel de dominio del pensamiento sistémico, I entre el segundo y tercer nivel de dominio del pensamiento sistémico; 0,83 I entre el primer y segundo nivel de dominio de la resolución de problemas.

Asimismo; 0,938 entre el segundo y tercer nivel de dominio de la resolución de problemas; 0,646 entre el primer nivel de dominio del pensamiento sistémico y la resolución de problemas; 0,934 entre el segundo nivel de dominio del pensamiento sistémico y la resolución de problemas; y 0,876 entre el tercer nivel de dominio del pensamiento sistémico y la resolución de problemas. 
Tabla 3. Causas de bajos logros en la resolución de problemas con el modelo constructivista.

\begin{tabular}{|c|c|}
\hline \multirow{5}{*}{ Académicas } & Desinterés de los estudiantes por participar en el desarrollo de la clase. \\
\hline & Prevalencia en los estudiantes del individualismo que el trabajo cooperativo. \\
\hline & Desistimiento por concebir un horizonte en común en el trabajo colaborativo. \\
\hline & Resistencia de los estudiantes para socializar los productos y resultados. \\
\hline & Poco conocimiento y manejo de los contenidos matemáticos. \\
\hline \multirow{4}{*}{ Materiales } & Aulas inadecuadas para las clases. \\
\hline & Textos escolares con predominio del conocimiento científico memorístico. \\
\hline & Situaciones problemáticas orientadas y cerradas a la práctica constructivista. \\
\hline & Cuaderno de trabajo con problemas ajenos a nuestro contexto. \\
\hline \multirow{8}{*}{ Metodológicas } & Evaluación centrada en la medición de conocimientos. \\
\hline & Omisión de la retroalimentación reflexiva. \\
\hline & Priorización y prevalencia del conductismo en la resolución de problemas. \\
\hline & Priorización por el desarrollo de algoritmos. \\
\hline & Ejecución didáctica centrada en el docente como expositor de conocimientos. \\
\hline & Desarrollo del enfoque de resolución de problemas solo con el constructivismo. \\
\hline & $\begin{array}{l}\text { Desconocimiento de la información necesaria relacionada con el modelo } \\
\text { constructivista. }\end{array}$ \\
\hline & $\begin{array}{l}\text { Resistencia docente por el desarrollo de estrategias de enseñanza-aprendizaje } \\
\text { centrado en la resolución de problemas. }\end{array}$ \\
\hline
\end{tabular}

En lo académico, las causas de los bajos logros en la resolución de problemas por parte de los estudiantes son: desinterés por participar en el desarrollo de la clase, prevalencia del individualismo que el trabajo colaborativo, desistimiento por concebir un horizonte en común en el trabajo colaborativo, resistencia para socializar los productos y resultados y poco conocimiento y manejo de los contenidos matemáticos.

En cuanto a las condiciones materiales las causas son: aulas inadecuadas para las clases, textos escolares con predominio del conocimiento científico y memorístico, situaciones problemáticas orientadas y cerradas a la práctica constructivista y cuadernos de trabajo con problemas ajenos a nuestro contexto.

Sobre los aspectos metodológicos las causas son: evaluación centrada en la medición de los conocimientos, omisión de la retroalimentación reflexiva, priorización y prevalencia del conductismo en la resolución de los problemas, priorización por el desarrollo de algoritmos, ejecución didáctica centrada en el docente como expositor de conocimientos, desarrollo del enfoque de la resolución de problemas solo centrado en el constructivismo, desconocimiento de la información relacionada con el modelo constructivista, resistencia docente por el desarrollo de estrategias de enseñanza-aprendizaje centrado en la resolución de problemas.

Se diseñó un modelo basado en el pensamiento sistémico para la resolución de problemas complejos de matemática. Sus componentes permiten desarrollar sesiones de aprendizaje en el que los estudiantes resuelven problemas complejos de matemática en el marco del diseño curricular nacional y los estándares de aprendizaje de la educación básica regular. 
Figura I. Modelo basado en el pensamiento sistémico para la resolución de problemas complejos de matemática.

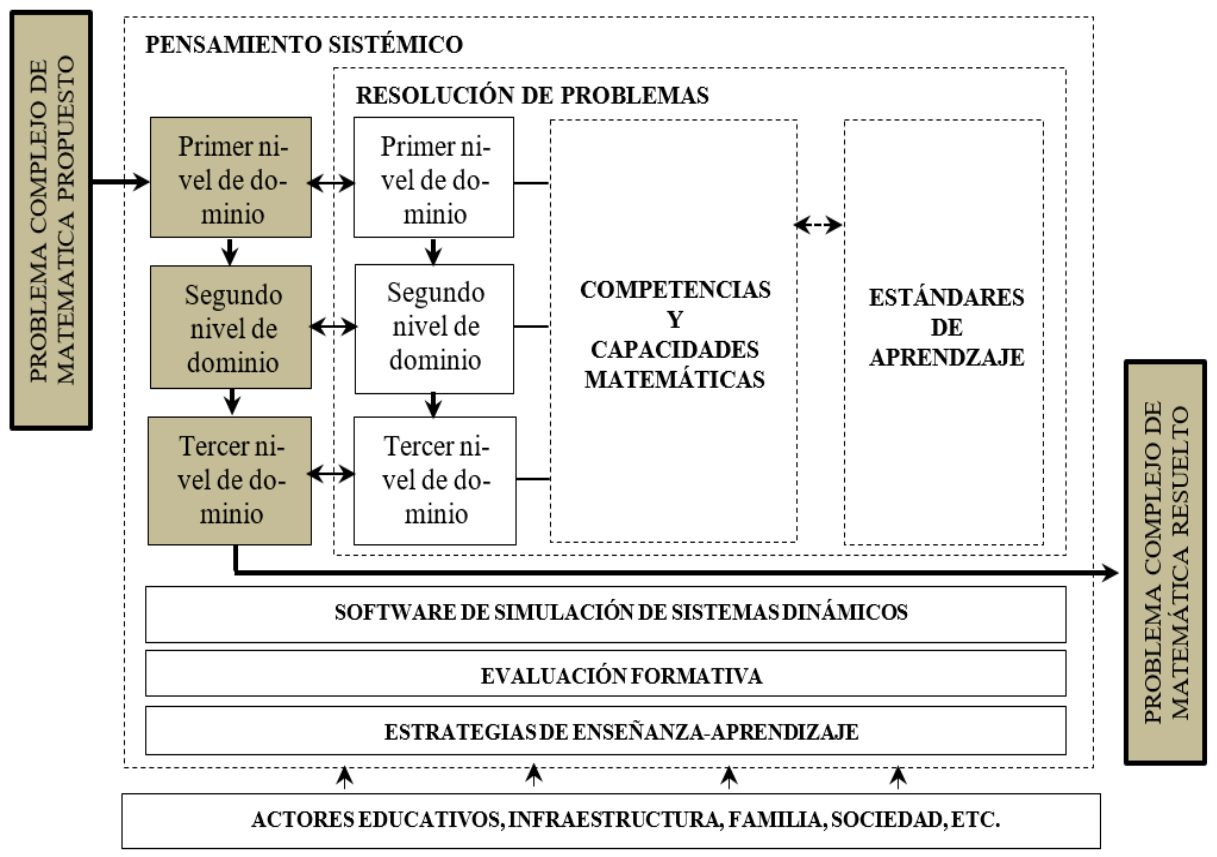

Figura 2. Metodología de aplicación práctica del modelo basado en el pensamiento sistémico para la resolución de problemas complejos de matemática en estudiantes del 3er grado de la I. E. "Santa Rosa".

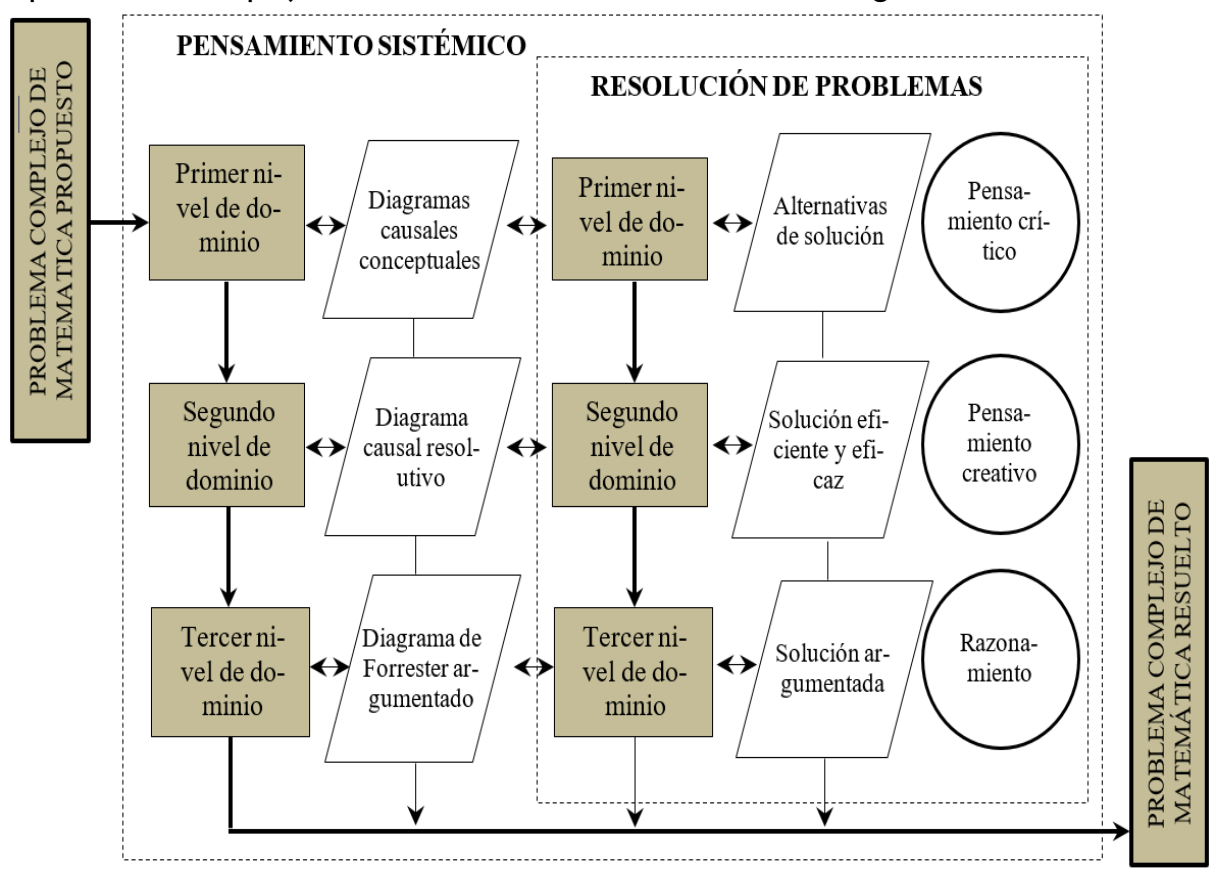

El pensamiento sistémico es el macrosistema del modelo con los componentes resolución de problemas, competencias y capacidades matemáticas, estándares de aprendizaje y estrategias de enseñanza-aprendizaje. Comprende la concreción de tres niveles de dominio, el primero inicia el proceso de resolución del problema complejo propuesto, el segundo permite decidir y consolidar la construcción de una solución eficiente y eficaz, y el tercero concreta la solución 
del problema complejo, resuelto con procedimientos formales simulados a través de un software de simulación dinámica.

La resolución de problemas constituye el mesosistema del modelo, que además de las competencias y capacidades matemáticas y los estándares de aprendizaje, concreta el desarrollo de sus tres niveles de dominio. En el primero se identifica y analiza el problema complejo planteado y propone alternativas de solución, en el segundo se analizan nuevas alternativas de solución y se construye una solución eficiente y eficaz, y en el tercero se propone una o más soluciones referidos a otros contextos o ámbitos de la realidad, relacionados con el problema complejo propuesto. Los niveles de dominio de la resolución de problemas se desarrollan simultáneamente a los del pensamiento sistémico.

El software de simulación de sistemas dinámicos es el componente tecnológico transversal que se utiliza desde el inicio de la resolución del problema e interviene en los tres niveles de dominio del pensamiento sistémico y de lo resolución de problemas, favorece en la intervención didáctica para lograr las competencias y capacidades del área de matemática, con la provisión de las herramientas del pensamiento sistémico que se emplean al resolver un problema complejo de matemática.

La evaluación formativa es un componente transversal que interactúa durante el proceso de resolución del problema complejo, interviniendo en todos los componentes del modelo. Permite la retroalimentación reflexiva durante el desarrollo de los niveles de dominio del pensamiento sistémico y de la resolución de problemas, a fin de corregir oportuna y recurrentemente los errores o inconvenientes en la aplicación del conocimiento matemático y los procedimientos didácticos soportados en diagramas causales y de Forrester.

Las estrategias de enseñanza-aprendizaje es un componente transversal a todo el proceso de la resolución del problema complejo, concebido en los enfoques del pensamiento sistémico y constructivismo. Para la planificación y ejecución se considera estrategias didácticas en contextos colaborativos, en el que se emplean a los diagramas causales como las herramientas del pensamiento sistémico, las estrategias de enseñanza-aprendizaje y los problemas contextuales reales e inmediatos a los estudiantes.

Como el modelo está basado en el pensamiento sistémico para la resolución de problemas dinámicos o complejos de matemática, está concebido como un sistema abierto en el que otros factores lo afectan. La influencia de otros sistemas o subsistemas externos, inciden en la intervención pedagógica que el docente realiza en el proceso de enseñanza de la resolución del problema complejo. Estos agentes externos son: los actores educativos, la infraestructura actual, las otras áreas del conocimiento, los recursos y materiales disponibles, y el contexto familiar y social de los estudiantes.

En cuanto al fundamento, la intervención didáctica planteada por Montenegro \& Schroeder (2020) en su investigación coincide con el modelo propuesto, dado que, el pensamiento sistémico es concebido como condición relevante para el aprendizaje y profundiza sus dimensiones: configuración del problema, abarcabilidad de factores, supuestos de causalidad y soportes simbólicos apelados; que tácitamente, también soportan la propuesta de enseñanza y aprendizaje de la resolución de problemas complejos de matemática de este estudio. El pensamiento sistémico se presenta como un patrón autoorganizado y se manifiesta como emergente interaccional y contextual más que como una competencia individual. 


\section{Discusión}

La alta y muy alta correlación entre las variables y subvariables del pensamiento sistémico (tablas I y 2) confirman la asociación directa entre ellas. A mayor dominio del pensamiento sistémico y de sus niveles, es también mayor el dominio de la resolución de problemas y de sus niveles; lo que garantiza un manejo adecuado de las herramientas del pensamiento sistémico. Lo encontrado coincide con Barragán et al. (2018) en lo referido a que, si el proceso de enseñanza es organizado correctamente y en forma sistemática en niveles progresivos de logro, garantiza el desarrollo del pensamiento complejo desde la sistematización de la enseñanza de la matemática.

Los bajos logros en la resolución de problemas de matemática en los estudiantes del tercer grado de educación secundaria de la I. E. "Santa Rosa", se deben a causas académicas, materiales y metodológicas (tabla 3). Corcino-Barrueta et al. (2021), enfatiza lo metodológico y manifiesta la necesidad de desarrollar procesos educativos de forma sistémica y holística, en el que la acción docente se integra al conjunto de todos los actores y recursos tangibles o no al acto de enseñar, y que son factores determinantes en el aprendizaje de conocimientos conceptuales, procedimentales y actitudinales utilizados en la resolución de problemas matemáticos, en combinación con herramientas del pensamiento sistémico.

El empleo inadecuado de un modelo de resolución de problemas centrado en el constructivismo sin el enfoque del pensamiento sistémico y sus herramientas, han conllevado a que lo estudiantes evidencien dificultades en la resolución de problemas. Al respecto, se ha propuesto un modelo (figuras I, 2) en el que el pensamiento sistémico se incorpora en las estrategias de enseñanza-aprendizaje para resolver de problemas complejos de matemática; Donoso Osorio et al. (2020) coinciden en considerar un enfoque holístico e integrador en los procesos crecientes en complejidad al resolver un problema, y propenden a potenciar la influencia de la intervención pedagógica sobre los logros de aprendizaje que se esperan.

El modelo propuesto recoge el aporte de los diagramas causales y el análisis en la complejidad para emplearla en las estrategias de enseñanza-aprendizaje al resolver el problema matemático, el cual favorece al desarrollo de la creatividad, como parte del pensamiento complejo; y al tomar en cuenta las herramientas que emplea el pensamiento sistémico, en el proceso de enseñanza y aprendizaje para la resolución de un problema matemático.

\section{Conclusión}

Existe una correlación $(\mathrm{t}=0,918)$ muy alta y positiva entre el pensamiento sistémico y la resolución de problemas matemáticos. Existen correlaciones muy altas y positivas del primer al segundo nivel de dominio $(t=l)$, y del segundo al tercer nivel de dominio $(t=l)$ del pensamiento sistémico. Existen correlaciones altas y positivas del primer al segundo nivel de dominio $(t=$ $0,831)$, y del segundo al tercer nivel de dominio $(t=0,938)$ de la resolución de problemas.

La correlación entre el primer nivel de dominio del pensamiento sistémico y de la resolución de problemas es moderada y positiva $(t=0,646)$. La correlación entre el segundo nivel de dominio del pensamiento sistémico y de la resolución de problemas es muy alta y positiva $(t=0,934)$. La correlación entre el tercer nivel de dominio del pensamiento sistémico y de la resolución de problemas es alta y positiva $(t=0,876)$. 
Las causas académicas que ocasionan bajos logros en la resolución de problemas matemáticos con el modelo constructivista son: el desinterés por participar en el desarrollo de las clases y la prevalencia del individualismo, el desistimiento por concebir un horizonte común de solución, la resistencia a la socialización de resultados; y el poco conocimiento y manejo de los contenidos matemáticos.

Las causas materiales que ocasionan bajos logros en la resolución de problemas matemáticos con el modelo constructivista son: el uso de textos escolares predominantemente memorísticos, el planteo de problemas orientados y cerrados solo a la práctica constructivista, y el empleo de cuadernos de trabajo con problemas ajenos al contexto.

Las causas metodológicas que ocasionan bajos logros en la resolución de problemas matemáticos con el modelo constructivista son: las evaluaciones que miden solo conocimientos y no considera a la retroalimentación reflexiva, la priorización y prevalencia del conductismo, la priorización de la práctica algorítmica, la ejecución didáctica centrada en el docente, el enfoque de resolución de problemas limitado al modelo constructivista, el desconocimiento de la información sobre el modelo constructivista, y la resistencia del docente por el desarrollo de estrategias de enseñanza-aprendizaje en el enfoque problémico.

El modelo basado en el enfoque del pensamiento sistémico en el que se emplean los diagramas causales conceptuales, diagramas causales resolutivos y diagramas de Fórrester argumentados; permiten la integración, articulación e interrelación con los componentes o elementos de la resolución de problemas de matemática y facilitan la solución de situaciones en contextos reales en la complejidad.

\section{Referencias bibliográficas}

Barragán Moyano, V. E., Jaque Sandoval, J. E., \& Acosta Patiño, E. I. (20I8). El pensamiento complejo desde la enseñanza de la Lógica Matemática (Revisión). Revista Científico Educacional De La Provincia Granma, 14(4), 169-181. https://revistas.udg.co.cu/index.php/roca/article/view/547

Corcino-Barrueta, F. E., Chamoli-Falcon, A. W., Otalora-Martinez, C. R., \& Melgarejo-Figueroa, M. D. P. (202I). El modelo sistémico de aprendizaje y enseñanza, como apoyo en la inserción laboral. Investigación Valdizana, 15(I), 31-40. https://doi.org/I0.33554/riv. I5.I.798

Donoso Osorio, E., Valdés Morales, R. A., Cisternas Núñez, P., \& Cáceres Serrano, P. (2020). Enseñanza de la resolución de problemas matemáticos: un análisis de correspondencias múltiples. Diálogos Sobre Educación, I I (2 I), I-22. https://doi.org/ 0.32870/dse.v0i2I.629

Gil Vera, V. D., \& Gil Vera, J. C. (2017). Seguridad informática organizacional: un modelo de simulación basado en dinámica de sistemas. Scientia et Technica, 22(2), 193-197. https://doi.org//0.225 I7/234472/4.II37|

Gutiérrez Verde, E., Rodríguez Ramos, P. A., \& Lavado Ruiz, C. (20I8). Mejoras para elevar la disponibilidad de las unidades acuáticas livianas. Ingenería Mecánica, 23(I), I-8. https://ingenieriamecanica.cujae.edu.cu/index.php/revistaim/article/view/62I

Ministerio de Educación. (2016). Educación Básica Regular. Programa Curricular de Educación 
Secundaria. Talleres gráficas del Minedu.

http://www.minedu.gob.pe/curriculo/pdf/03062016-programa-nivel-secundaria-ebr.pdf

Montenegro, G., \& Schroeder, I. (2020). Dimensiones del pensamiento sistémico aplicado: un estudio de casos múltiple desde la perspectiva de sistemas complejos y el aprendizaje organizacional. Psicología, Conocimiento y Sociedad, 10(2), 74-100. https://doi.org/I0.26864/PCS.v10.n2.4

Sáenz López, K., \& Tamez González, G. (2015). Métodos y técnicas cualitativas y cuantitativas aplicables a la investigación en ciencias sociales (I st ed.). Tirant lo Blanch.

Villa Sánchez, A., \& Poblete Ruiz, M. (20I3). Aprendizaje basado en competencias. Una propuesta para la evaluación de las competencias genéricas (3rd ed.). In E. Mensajero.

\section{Financiamiento}

Ninguno.

\section{Conflicto de intereses}

El artículo no presenta conflicto de intereses.

\section{Contribución de autores}

Montilla-García, Henrry: Investigador y redactor del artículo. 